\title{
Overview of Transmission Characteristics in Novel Wireless Underground Sensor Networks
}

\author{
Xiaoqing Yu, Wenting Han and Zenglin Zhang \\ Northwest A \& F University, Shaanxi, Yangling, 712100, China \\ Research Institute of Water-saving Agriculture of Arid Regions of China, Shaanxi, \\ Yangling, 712100, China \\ yuxiaoqing115@gmail.com,wentinghan200@126.com, \\ zhangzenglin115@gmail.com
}

\begin{abstract}
Many applications for agricultural information and environment monitoring exploit buried sensors wired-connected to the soil surface for information retrieval. A novel wireless underground sensor networks is a specialized kind of wireless sensor network that communicates through soil. The wireless underground sensor networks (WUSN) consist of wireless devices that are buried completely under dense soil and operate below the ground surface. In this paper, the application research of the wireless underground sensor networks is described. Experimental measurements of the signal strength and packet error rate are presented at the frequency of $433 \mathrm{MHz}$, which show a good agreement with the theoretical studies. The results show the buried depth of the WUSN node and the volumetric water content of the soil are the most important factor to adversely affect the communication.
\end{abstract}

Keywords: Wireless sensor networks, agricultural, information, communication, acquisition

\section{Introduction}

At present, the wireless sensor network for agricultural information acquisition belongs to the terrestrial wireless sensor network system. The sensor network usually connects sensors to ground data reading and wireless transceiver equipment through cable way in order to avoid the communication in underground soil [1-5]. These equipment exposure on the ground that not only influence farming, wireless transmitting function of wireless node also can be seriously affected because of the geography, weather and other natural factors. Based on these disadvantages, a kind of wireless underground sensor network provides a new way for underground information monitoring, and also become the research direction for agricultural information collection technology [6].

Wireless underground sensor networks (WUSN) is that the sensor equipment with wireless transceiver module buried completely in certain soil depth, sensor module percepts the data and send data through the wireless mode[7,8]. Many sensor nodes consist of network in the soil and complete automatically the process of data perception, collection. The WUSN has many advantages, such as strong concealment, easy of deployment, timeliness of the data, reliability, large covering range, easy of upgrade, etc [6]. Besides monitoring of soil static parameters, wireless underground sensor network can also be used for monitoring of soil movement situation, forecast of the earthquake, landslide, underground ice motion and volcanic eruptions, so it has broad application prospects in agriculture, military, transportation, construction, natural disaster forecast etc., [9-12].

The rest of this paper is organized as follows: In Section 2, the related work of the electromagnetic wave communication in the soil is given. In Section 3, the test materials 
for the experiments and the experimental methodology are described. The experiment results for the communication of wireless underground sensor networks internodes are presented. Finally, summary and future plan for continuation of this work are discussed in Section 5.

\section{Related Work}

Wireless underground sensor networks have been investigated in many contexts recently, but research reports of wireless underground sensor networks in agricultural application are little. The concept of WUSN and the challenges related to the underground wireless channel have been introduced in [6]. The near surface wireless underground sensor networks system used for golf course was developed and the acquisition nodes, relay nodes and gateway node were designed in [13].

In [14], wireless signal attenuation of ZigBee wireless transceiver module of the 2.44 $\mathrm{GHz}$ frequency was researched by using soil column in different soil types and the water content. Experimental results showed that increase of soil column depth and volumetric water content of the soil could lead to increase of signal attenuation, the relationship could be expressed in linear model, and the correlation coefficient R2 is greater than 0.90 . In [15], there had been found that rainfall and stormy weather environmental conditions, and the soil compactness, soil density and vegetation cover degree, topology structure parameters of wireless underground sensor networks, sampling time and sampling density had great influence on the distortion degree of the soil moisture acquisition signal. In [16], Angelo R. and Silva studied the influence of the communication performance between the terrestrial nodes and the underground nodes in some factors, including antenna bandwidth of WSN nodes in $433 \mathrm{MHz}$ frequency, the buried depth of nodes in the soil $(15 \mathrm{~cm}$ and 35 $\mathrm{cm}$ ) and water content of the soil (volumetric water content was $9.5 \%$ and $37.3 \%$, respectively).

\section{Materials and Methodology}

The underground experiments with $433 \mathrm{MHz}$ sensor nodes were carried out in the laboratory of the Research Institute of Water-saving Agriculture of Arid Regions of China in the Northwest Agriculture and Forestry University. In the trial, we measure the clay percent as $11.32 \%$, the silt percent as $61.26 \%$, the sand particle percent as $27.42 \%$, and the solid soil particle density as $2.6 / \mathrm{cm} 3$ unless otherwise noted. The basic physical property index of the soil sample is shown in Table 1. The results are presented considering how some important parameters affect the wireless underground sensor networks communication: the burial depth of WUSN node and volumetric water content of the soil.

Table 1. The Basic Physical Property Index of the Soil Sample

\begin{tabular}{cccc}
\hline \multirow{2}{*}{ Soil type } & \multicolumn{3}{c}{ Particle-sized fractions (\%) } \\
\cline { 2 - 4 } & Sand & Silt & Clay \\
Silty & 27.4 & & \\
loam & 2 & 61.26 & 11.32 \\
\hline
\end{tabular}

For the experiments, WUSN nodes use $433 \mathrm{MHz}$ band. The output power were always set to maximum transmit power of $20 \mathrm{dBm}$. The antenna of sensors nodes are a standard one-quarter wavelength monopole antenna with $17 \mathrm{~cm}$ lengths, and the antennas are vertically oriented. The tests were designed to collect the received signal strength and error rate of communication. Each experiment in this work is based on a set of 2 experiments with 500 messages, which result in a total of 1000 packets. The number of packets correctly received by one or more receiver nodes is recorded along with the signal 
strength for each packet. Accordingly, the packet error rate and the received signal strength level from each receiver are collected. To prevent the effects of hardware failures of each individual node, qualification tests have been performed before each experiment.

\section{Experimental Results and Discussion}

In the paper, the received signal strength and the packet error rate of electromagnetic wave transmission in the soil medium are studied under $433 \mathrm{MHz}$ frequency. The effects of node burial depth, the horizontal inter-node distance and volumetric water content of the soil parameters on received signal strength and packet error rate are established through Mat lab in the wireless underground sensor networks communication.

\subsection{Effects of Node Burial Depth and Soil Moisture}

To investigate the effects of node burial depth and volumetric water content of the soil on the signal strength and packet error rate, the horizontal inter-node distance between the sender and the receiver is fixed $50 \mathrm{~cm}$. In the communication between underground nodes, the sender node is fixed depth $40 \mathrm{~cm}$, the receiver node is varied from $10 \mathrm{~cm}$ to $100 \mathrm{~cm}$. Moreover, the range of the soil volumetric water content of the soil is 5\%-30\% in the underground-to-underground communication. In Figure 1 and Figure 2, the received signal strength and packet error rate values are shown, respectively, as a function of the receiver node depth and volumetric water content of the soil.

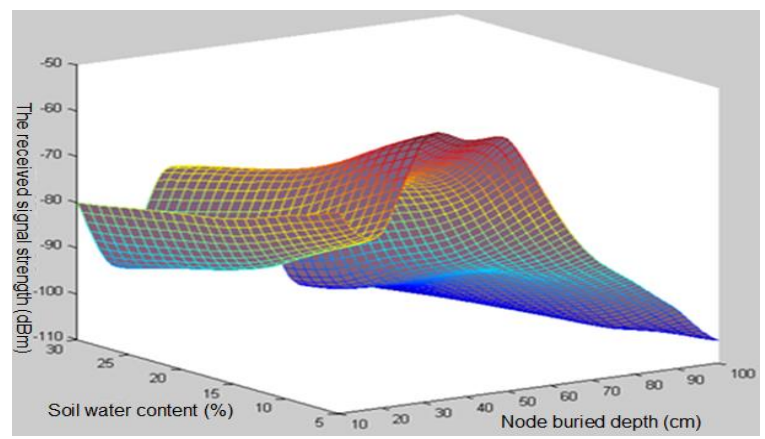

\section{Figure 1. Tests for the Received Signal Strength. The Underground Node is varied from $10 \mathrm{~cm}$ to $100 \mathrm{~cm}$}

The experiments show that a higher burial depth and volumetric water content of the soil also implies an increase in the soil path, higher attenuation is observed in the WUSN underground to underground communication. It can be observed in Figure 1, that received signal strength gradually reduced with the increase of the receiver node depth when WUSN sender node buried depth keeps in depth $40 \mathrm{~cm}$. When receiver node buried depth range $30 \mathrm{~cm}-50 \mathrm{~cm}$, received signal strength reaches maximum. Received signal strength gradually increases when receiver node buried depth is less than $30 \mathrm{~cm}$ and change to 10 $\mathrm{cm}$, received signal strength gradually decreases when receiver node buried depth is more than $50 \mathrm{~cm}$. In the communication of underground sensor internodes, the sender node energy is increased because of the reflection wave that electromagnetic wave penetrates the ground and reflects back in the top soil, so the receiver node received signal strength of receiver node becomes higher. Therefore, in the same actual distance and horizontal internodes distance of WUSN nodes, the received signal strength is not the same if the nodes burial depth is different.

In addition, received signal strength shows a trend of decrease with the increase of soil volumetric water content. From Figure 1, we can conclude that soil volumetric water content is less than $30 \%$ when receiver node buried depth keep in the whole range, the minimum received signal strength is higher than $-110 \mathrm{dBm}$. The received signal strength 
reaches the minimum when the soil volumetric water content is $30 \%$. In the communication of WUSN underground sensor internodes, with the increase of soil volumetric water content interval 5\%, received signal strength reduces $2 \mathrm{dBm}-10 \mathrm{dBm}$ when the receiver node buried depth below $80 \mathrm{~cm}$. The decrease extent of the received signal strength becomes small when receiver node buried depth is higher than $80 \mathrm{~cm}$, average $1 \mathrm{dBm}$. So soil volumetric water content is also an important factor that influences received signal strength, when the soil moisture content is larger, the electromagnetic wave propagation signal attenuation is also larger, and the received signal strength is relative smaller.

Moreover, the analysis is carried on through Matlab, relationship is established in Equation 1.

$$
\begin{gathered}
R_{s s}=-53.8017+0.2083 N_{d}-1.8269 S_{v}-0.0065 N_{d}^{2}+0.0048 N_{d} S_{v}+0.0231 S_{v}{ }^{2} \\
R^{2}=0.814
\end{gathered}
$$

where Rss is the received signal strength, $\mathrm{dBm}$; $\mathrm{Nd}$ is the underground node burial depth, cm; $\mathrm{Sv}$ is volumetric water content of the soil,\%。 It can be seen from the equation 1 , the changes of the node burial depth $\mathrm{Nd}$ and volumetric water content of the soil Sv have binary quadratic relationship on the received signal strength Rss in the wireless underground communication, goodness-of-fit R2 is higher.

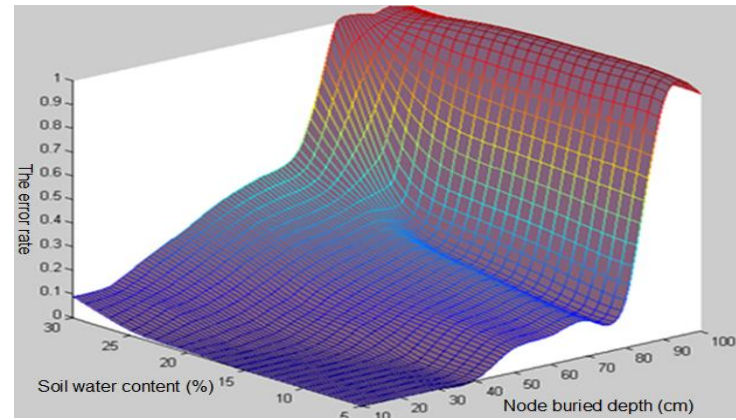

\section{Figure 2. Tests for Packet Error Rates. The Underground Received Node is Varied from $10 \mathrm{~cm}$ to $100 \mathrm{~cm}$}

It can be included in Figure 2, that communication error rate gradually increases with the receiver node buried depth and soil moisture content change in the circumstances of underground sensor sender node buried depth and the horizontal inter-node distance remain the same, the maximum error rate reaches $100 \%$. Furthermore, error rate increases sharply to maximum $100 \%$ when soil volumetric water content range is $5 \%-25 \%$ and WUSN underground receiver node buried depth $90 \mathrm{~cm}$. Another, the error rate increases with the increase of soil volumetric water content in the same receiver node burial depth, the maximum increase extent $15 \%$. Therefore, the error rate of the underground internodes communication is quite different if soil volumetric water content is different.

Moreover, it can be observed in Figure 2, that WUSN node electromagnetic wave communication produces the error rate in the underground soil that increases with the increase of soil volumetric water content, when the soil volumetric water content change in the range of 5\%-30\% and receiver node buried depth $10 \mathrm{~cm}-70 \mathrm{~cm}$, but the biggest error rate is no more than 50\%. The communication error rate of WUSN underground sensor internodes increases with the increase of soil volumetric water content, when the receiver node buried depth $80 \mathrm{~cm}$ and soil volumetric water content change from 5\% to $25 \%$, the maximum error rate is about $40 \%$.

The analysis is carried on through Matlab, relationship is established in Equation 2. 


$$
\begin{gathered}
E_{r}=0.1313-0.0098 N_{d}-0.0101 S_{v}+0.0002 N_{d}{ }^{2}+0.0005 S_{v}{ }^{2} \\
R^{2}=0.8845
\end{gathered}
$$

where $\mathrm{Er}$ is the packet error rate; $\mathrm{Nd}$ is the underground node burial depth,cm; $\mathrm{Sv}$ is volumetric water content of the soil,\%。 It can be seen from the equation 2; the changes of the node burial depth $\mathrm{Nd}$ and volumetric water content of the soil Sv have binary quadratic relationship on the error rate $\mathrm{Er}$ in the wireless underground communication, goodness-of-fit R2 is higher.

\subsection{Effects of the Horizontal Inter-Node Distance and Soil Moisture}

In the propagation process of wireless underground sensor network electromagnetic wave in the soil medium, underground sensor internodes communication changes with the horizontal inter-node distance change. Therefore, WUSN horizontal inter-node distance $n$ is also one of the important factors that influence communication. In the test of WUSN node underground to underground communication, the sensor node RF frequency uses $433 \mathrm{MHz}$, underground sensor sender and receiver node buried depth are fixed $40 \mathrm{~cm}$, soil volumetric water content change in the range of $5 \%$ to $30 \%$, the change range of horizontal inter-node distance is $10 \mathrm{~cm}$ to $100 \mathrm{~cm}$. In this condition, effects of horizontal inter-node distance and soil moisture content on received signal strength and the error rate are measured in the underground sensor internodes communication, as shown in Figure 3 and Figure 4.

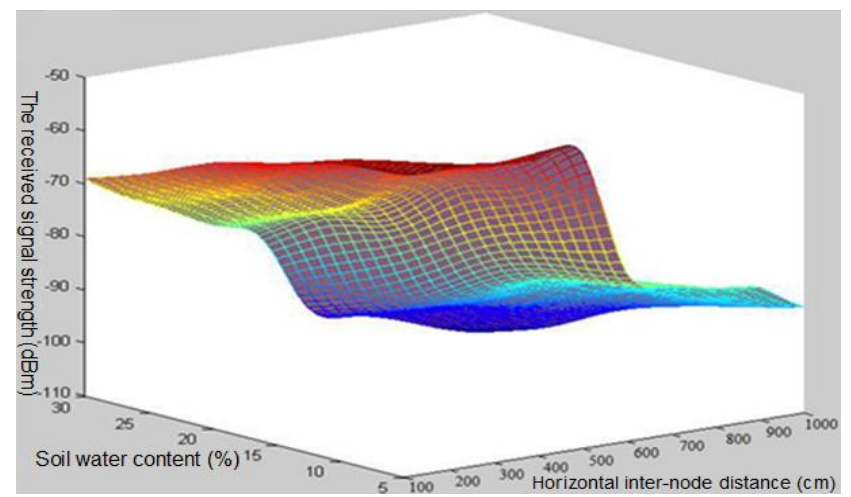

\section{Figure 3. Tests for the Received Signal Strength. The Horizontal Inter-Node Distance is Varied from $10 \mathrm{~cm}$ to $100 \mathrm{~cm}$}

It can be showed in Figure 3, the received signal strength changes with the change of the horizontal inter-node distance and soil moisture content when WUSN sender and receiver node buried depth are fixed in $40 \mathrm{~cm}$. As shown in Figure 3, the received signal strength decreases with the increase of the horizontal inter-node distance when soil volumetric water content remains the same circumstances. In addition, we can conclude that the received signal strength decrease with the increase of horizontal inter-node distance and soil moisture content in the underground sensor internodes communication when the horizontal inter-node distance change in $10 \mathrm{~cm}-100 \mathrm{~cm}$ range and soil moisture content is less than $25 \%$, the minimum received signal strength is not less than $-100 \mathrm{dBm}$. The received signal strength gradually decreases with the increase of the horizontal internode distance when the soil moisture content is $25 \%$, the minimum value is about -105 $\mathrm{dBm}$. When soil moisture content increase continually to $30 \%$, the received signal strength reduces with the increase of horizontal inter-node distance and reaches minimum, about $-110 \mathrm{dBm}$.

Figure 3 shows that the received signal strength decreases with the increase of the soil moisture content in the process of WUSN node communication, when the horizontal 
inter-node distance changes in the whole change range and soil moisture content change in the range of $5 \%$ to $15 \%$, about $3 \%$. When soil moisture content reaches $20 \%$, the received signal strength value reaches $-100 \mathrm{dBm}$ in the most horizontal inter-node distance $100 \mathrm{~cm}$. Soil moisture content increases continually to $25 \%$, the received signal strength is $-100 \mathrm{dBm}$ when the horizontal inter-node distance is $80 \mathrm{~cm}$. After that, the horizontal inter-node distance continues to increase, the received signal strength decreases constantly till the minimum about $-105 \mathrm{dBm}$. The received signal strength value achieves $-100 \mathrm{dBm}$ when the soil moisture content is the maximum $30 \%$ and the horizontal internode distance is $60 \mathrm{~cm}$. Then, the received signal strength decreases gradually with the increase of the horizontal inter-node distance till the minimum value achieves $-110 \mathrm{dBm}$, decrease extent is $2 \mathrm{dBm}-3 \mathrm{dBm}$. So, in the WUSN underground sensor internodes communication, the horizontal inter-node distance and soil moisture content are the biggest factors that influence the received signal strength.

Moreover, the analysis is carried on through Matlab, relationship is established in Equation 3.

$$
\begin{gathered}
R_{s s}=-37.8433-0.0502 H_{s}-0.5746 S_{v}-0.0103 S_{v}{ }^{2} \\
R^{2}=0.9449
\end{gathered}
$$

where Rss is the received signal strength, $\mathrm{dBm}$; $\mathrm{Hs}$ is the horizontal inter-node distance, $\mathrm{cm} ; \mathrm{Sv}$ is volumetric water content of the soil,\%。 It can be seen from the equation 3 , the changes of the horizontal inter-node distance $\mathrm{Hs}$ and volumetric water content of the soil Sv have binary quadratic relationship on the received signal strength Rss in the wireless underground communication, goodness-of-fit R2 is higher.

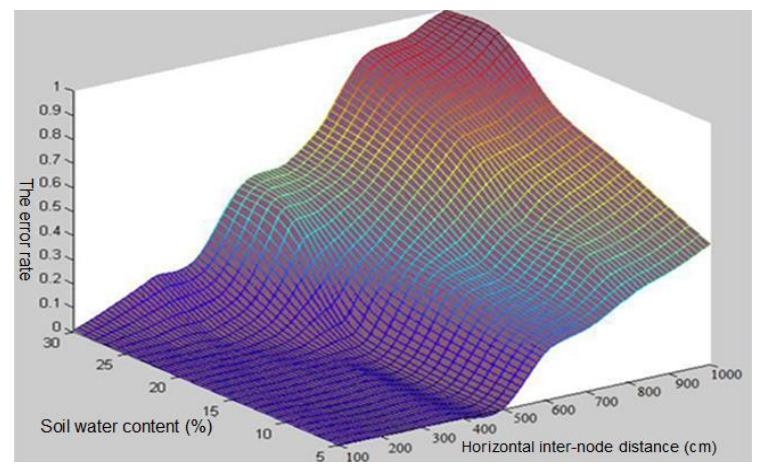

\section{Figure 4. Tests for Packet Error Rates. The Horizontal Inter-Node Distance is varied from $10 \mathrm{~cm}$ to $100 \mathrm{~cm}$}

It can be observed in Figure 4, that WUSN underground to underground communication error rate increases gradually with the increase of the horizontal internode distance and soil moisture content, the maximum error rate reaches $100 \%$. We can include from Figure 4, the error rate increases gradually when the soil moisture content is less than $25 \%$ and the horizontal inter-node distance changes in the whole range of $10 \mathrm{~cm}$ $100 \mathrm{~cm}$. At the maximum horizontal inter-node distance, the error rate increase extent is almost equal to $10 \%$ with soil moisture content change, but the biggest error rate is no more than $80 \%$. When soil moisture content is more than $25 \%$, the error rate reaches $100 \%$ in the maximum horizontal inter-node distance.

In addition, Figure 4 shows that the error rate rises sharply at the horizontal inter-node distance $60 \mathrm{~cm}$, when soil moisture content changes in the range of $5 \%$ to $20 \%$, about $20 \%-30 \%$. Then, the error rate increases gradually with the increase of the horizontal inter-node distance. In the WUSN sensor node underground to underground communication, the error rate rise extent increases sharply when soil moisture content is more than $25 \%$ ant the sensor node horizontal distance is $50 \mathrm{~cm}$, about $30 \%$. After that, 
the error rate also changes gradually with the horizontal inter-node distance increase gradually, to maximum $100 \%$. In a word, sensor nodes are buried in soil in the process of WUSN underground to underground communication, electromagnetic wave communicates completely through the soil medium. Sensor node burial depth, the horizontal inter-node distance and soil moisture content are important influence factor in communication.

Moreover, the analysis is carried on through Mat lab; relationship is established in Equation 4.

$$
\begin{gathered}
E_{r}=0.0346-0.0003 H_{s}-0.0113 S_{v}+0.0003 S_{v}{ }^{2} \\
R^{2}=0.9630
\end{gathered}
$$

where $\mathrm{Er}$ is the packet error rate; $\mathrm{Hs}$ is the horizontal inter-node distance, $\mathrm{cm} ; \mathrm{Sv}$ is volumetric water content of the soil, $\%$ 。 It can be seen from the equation 4 , the changes of the horizontal inter-node distance $\mathrm{Hs}$ and volumetric water content of the soil Sv have binary quadratic relationship on the error rate $\mathrm{Er}$ in the wireless underground communication, goodness-of-fit R2 is higher.

\section{Conclusions}

Wireless underground sensor networks are a promising new and the main technical means in the collection of agricultural information. In this work, we propose the application of wireless underground sensor networks and present experiment results of underground to underground communication for WUSN. The experiment results reveal that the feasibility of WUSN communication with $433 \mathrm{MHz}$ frequency. Moreover, the experiment results show that the burial depth of WUSN node is important for the WUSN tests due to the attenuation of soil medium. In addition, we have shown that the horizontal inter-node distance plays an important role in the communication of WUSN. Finally, the direct influence of volumetric water content of the soil on the communication success is shown.

\section{Acknowledgements}

The authors wish to thank the National Engineering Research Center for Water-Saving Irrigation, which partially supported this research through the "China Postdoctoral Science Foundation funded project"(2014M552495) and the "Twelfth Five-Year" National Science and Technology Support Program" (2011BAD29B08), The authors are also grateful to the anonymous reviewers for their valuable feedback.

\section{References}

[1] Z. Butler and D. Rus, "Event-based Motion Control for Mobile Sensor Networks", IEEE Pervasive Computing, vol. 2, (2003), pp.34-43.

[2] Z. Abrams, A. Goel and S. Plotkin, "Set K-Cover Algorithms for Energy Efficient Monitoring in Wireless Sensor Networks", Proceedings of the 3rd International Conference on Information Processing in Sensor Networks, (2004).

[3] M. K. Watfa, H. Al-Hassanieh and S. Salmen, "A novel solution to the energy hole problem in sensor networks", Journal of network and computer applications, vol. 36, no. 2, (2013), pp.949-958.

[4] J. Carle and D. SimPlot-Ryl, "Energy Efficient Area Monitoring by Sensor Networks", IEEE Computer Magazine, vol. 37, (2004), pp.40-46.

[5] D. Hoplaros, Z. Tari and I. Khalil, "Data summarization for network traffic monitoring", Journal of network and computer applications, vol. 37, no. 1, (2014), pp.194-205.

[6] I. F. Akyildiz and E. P. Stuntebeck, "Wireless underground sensor networks: Research challenges", Ad Hoc Networks, vol. 4, (2006), pp.669-686.

[7] I. F. Akyildiz, W. Su, Y. Sankarasubramaniam and E. Cayirci, "Wireless Sensor Networks: A Survey", Computer Networks, no. 38, (2002), pp.393-422. 
[8] I. F. Akyildiz, M. C. Vuran and Z. Sun, "Channel modeling for Wireless Underground Communication in Soil”, Physical Communication, (2009).

[9] P. Erich, P. D. Stuntebeck and T. Melodia, "Wireless Underground Sensor Networks using Commodity Terrestrial Motes", Wireless Mesh Networks, (2006), pp.112-114.

[10] L. Li and X. M. Wen, "Energy Efficient Optimization of Clustering Algorithm in Wireless Sensor Network", Journal of electronics \& information technology, no. 30, (2008), pp.966-969.

[11] L. Li, H. X. Li and H. Liu, "Greenhouse Environment Monitoring System Based on Wireless Sensor Network", Transactions of the Chinese Society for Agricultural Machinery, vol. 9, (2009), pp.228-231.

[12] O. Green, E. S. Nadimi and V. Blanes, "Monitoring and modeling temperature variations inside silage stacks using novel wireless sensor networks", Computers and Electronics in Agriculture, vol. 69, no.1, (2009), pp.149-157.

[13] C. J. Ritsema, K. Henk and K. Leon, "A new wireless underground network system for continuous monitoring of soil water contents", Water resources research, vol. 45, (2009), pp.36-44.

[14] H. R. Bogena, J. A. Huismana, H. Meierb, U. Rosenbauma and A. Weuthena, "Hybrid wireless underground sensor networks", Quantification of signal attenuation in soil, Vadose Zone Journal, vol. 8, (2009), pp.755-761.

[15] D. Xin and M. C. Vuran, "Spatio-temporal Soil Moisture Measurement with Wireless Underground Sensor Networks", Ad Hoc Networking Workshop, (2010).

[16] A. R. Silva and M. C. Vuran, "Communication with above devices in wireless underground sensor networks: a empirical study”, 2010 IEEE International Conference Proceedings, (2010), pp. 23-27.

\section{Authors}

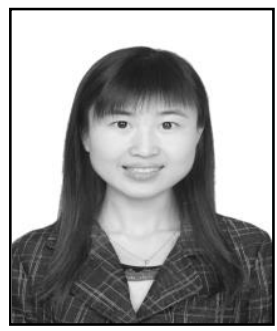

Xiao Q. Yu, received the B.S. degree from Department of Information Engineering, Lanzhou University of Finance and Economics, Lanzhou, China in 2006. She received her M.S. degree and Ph.D. degree from Department of Mechanical and Electric Engineering and Department of Water Resources and Architectural Engineering, Northwest A \& F University, Shaanxi, China in 2009 and 2013, respectively. Currently, she is pursuing post doctor degree from Department of Water Resources and Architectural Engineering under the supervision of Prof. Wen T. Han. Her current research interests are in Agricultural Water-Soil Engineering and Wireless Sensor Networks.

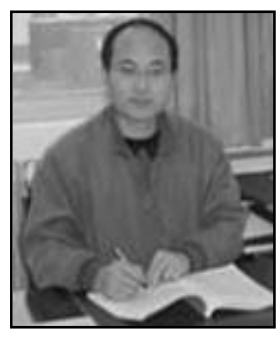

Wen T. Han, received his B.S. degree from Department of Mechanical and Electric Engineering, Northwest Agriculture University, Shaanxi, China in 1996. M.S. and Ph.D. degree from Department of Mechanical and Electric Engineering, Northwest A \& F University, Shaanxi, China in 1999 and 2004, respectively.

\section{Working Experiences:}

2005-present: researcher, Institute of Soil and Water Conservation of Chinese Academy of Sciences Northwest A \& F University, National Engineering Research Center for Water Saving Irrigation at Yangling.

2004-2005: Assistant Professor, Department of Mechanical and Electric Engineering, Northwest A \& F University.

2001-2004: A lecturer, Department of Mechanical and Electric Engineering, Northwest A \& F University.

\section{Research Interests:}

Information monitoring of crop and environment; intelligent control for precise irrigation; water distribution Simulation of sprinkler irrigation; Development of nozzle

Currently, he published academic papers more than 30 , including SCI and EI articles 16; the national invention patent 6. 


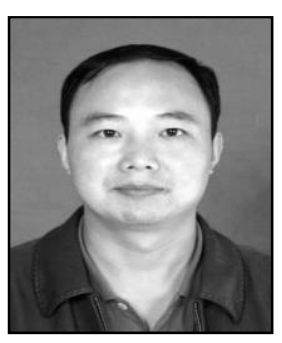

Zeng L. Zhang received his B.S. degree from Department of Mechanical and Electric Engineering, Harbin institute of Technology, Harbin, and M.S. degree from Department of Mechanical and Electric Engineering, Northwest A \& F University, Shaanxi, China in 2000 and 2007, respectively.

Currently, he is a teacher in Department of Mechanical and Electric Engineering, Northwest A \& F University, Shaanxi. He is pursuing Ph.D. degree under the supervision of Prof. Pu T. Wu. His current research interests are in Agricultural Water-Soil Engineering and Wireless Sensor Networks. 
International Journal of Future Generation Communication and Networking Vol. 8, No. 2 (2015) 